\title{
Hypercoagulable Thrombophilic Defect and Hyperhomocysteinemia with Recurrent Pregnancy Loss
}

\author{
${ }^{1}$ PG Rooplata, ${ }^{2} \mathrm{R}$ Nagarathnamma, ${ }^{3}$ Nagendra Prasad
}

\begin{abstract}
Successful fetal outcome in any pregnancy is dependent on adequate placental circulation. Normal physiological changes in pregnancy produce a hypercoagulable state.

Placental vasculature abnormalities may result in a number of gestational defects. They also can cause loss of pregnancy, intrauterine fetal death, intrauterine growth retardation, placental abruption, and preeclampsia.

Hereditary thrombophilias are usually undiagnosed because most carriers are asymptomatic. Placental perfusion may be compromised by increased thrombosis that leads to pregnancy complications and recurrent pregnancy loss (RPL).

We report a case of hypercoagulable thrombophilic defect and hyperhomocysteinemia with RPL.
\end{abstract}

Keywords: Hyper-homocysteinemia, Pregnancy loss, Thrombophilia.

How to cite this article: Rooplata PG, Nagarathnamma R, Prasad N. Hypercoagulable Thrombophilic Defect and Hyperhomocysteinemia with Recurrent Pregnancy Loss. J Med Sci 2016;2(3):50-52.

Source of support: Nil

Conflict of interest: None

\section{INTRODUCTION}

Recurrent pregnancy wastage is a major health problem that affects up to $5 \%$ of women of reproductive age. Known etiologic factors like chromosomal defects, uterine deformities, endocrine dysfunction, such as hypothyroidism, luteal phase inadequacy, and infectious disorders account for only about one-third of all pregnancy loss. ${ }^{1}$

The risk of venous thromboembolic events is high during pregnancy due to both physiologic changes of pregnancy and the additional impact of the inherited and acquired thrombophilias. Rate of venous thromboembolic events in pregnancy is 200 per 100,000 deliveries. The main

${ }^{1}$ Resident, ${ }^{2}$ Professor, ${ }^{3}$ Associate Professor

${ }^{1-3}$ Department of Obstetrics and Gynecology, RajaRajeswari Medical College \& Hospital, Bengaluru, Karnataka, India

Corresponding Author: Nagendra Prasad, Associate Professor Department of Obstetrics and Gynecology, RajaRajeswari Medical College \& Hospital, Bengaluru, Karnataka, India, Phone: +919901499440, e-mail: drnnprasad@gmail.com risk appears to occur in the postpartum period where the incidence increases almost 2.5-fold and is estimated at 500 per 100,000. A successful outcome of pregnancy requires an efficient uteroplacental vascular system since this system may be compromised by disorders of hemostasis associated with prothrombotic state. Inherited thrombotic defects and hyperhomocysteinemia are associated with thrombosis and recurrent pregnancy loss (RPL). Hereditary thrombophilias are usually undiagnosed because most carriers are asymptomatic; placental perfusion may be compromised by increased thrombosis that leads to pregnancy complications and RPL.

\section{CASE REPORT}

A 24-year-old resident of Bengaluru, belonging to low socioeconomic status, came with gravida 4 , para 3 with no living children, and presented at 6 weeks of gestation, for a routine antenatal checkup. Patient was investigated for RPL and was found to have deficiency of antithrombin (AT) III, and the hyperhomocysteinemia patient was followed up until term.

\section{Investigations}

Complete blood count, oral glucose challenge test, thyroid function test, prothrombin time, and activated partial thromboplastin time were normal. Homocysteine level was $22.84 \mu \mathrm{mol} / \mathrm{L}$; AT III was $21.3 \mathrm{mg} / \mathrm{dL}$; toxoplasmosis, rubella, cytomegalovirus, and herpes was negative; and anticardiolipin antibodies and lupus anticoagulant were negative.

\section{TREATMENT}

Patient was started on

- Low-molecular-weight heparin (enoxaparin $40 \mathrm{mg}$ subcutaneous once a day)

- Ecosprin $75 \mathrm{mg}$ once a day

- Tab homocyst (choline bitartrate + folic acid + methylcobalamine + vitamin $B_{6}$ ); patient was followed up until term.

Antenatal period was uneventful. Heparin was stopped day before the surgery and the baby was delivered at 38 weeks by elective lower (uterine) segment 


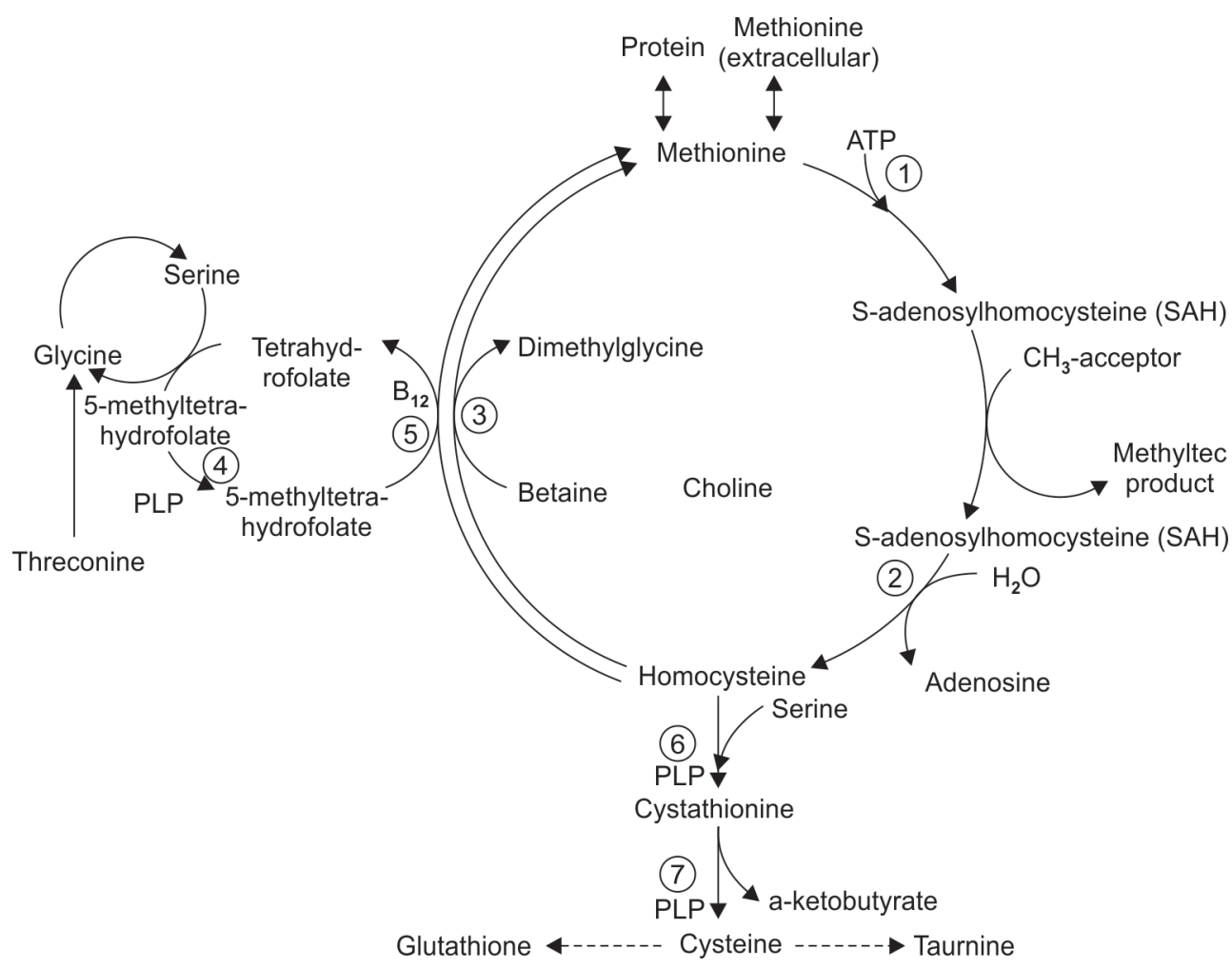

Fig. 1: Methionine cycle showing production of homocysteine

cesarean section in view of RPL; the postoperative period was uneventful for mother and baby.

\section{DISCUSSION}

Thrombophilias have increased risk of thrombosis and are at high risk for fetal loss, stillbirths, and possibly other serious obstetric complications. Though treatment strategies are still obscure in inherited thrombophilias as they are rarely seen in arterial thrombosis, thorough examination of the young patients with inherited thrombophilia should be considered. Treatment primarily involves a rapidly acting anticoagulant, such as heparin or lowmolecular-weight heparin and long-term anticoagulation with warfarin may be instituted to prevent recurrence. Thrombophilia is found in the majority of women with idiopathic pregnancy loss and is associated with late pregnancy wastage. These findings of pregnancy loss have paved the way for studies designed to prevent miscarriages by administering antithrombotic therapy. Early reports have suggested that antithrombotic therapy with low-molecular-weight heparin may result in improved fetal outcome in women with thrombophilia. ${ }^{2}$

Therapy with fibrinolytics is not used regularly because of the risk of serious bleeding and other complications. Our case was followed up with a low-molecularweight heparin during pregnancy and no complications were noted. Therefore, accurate treatment and regular follow-up must be carried out throughout the pregnancy. Thrombophilias are well known to cause RPL ${ }^{1-3}$; in view of this, the patient was managed accordingly and successful pregnancy was obtained in this case.

\section{HOMOCYSTEINE}

Homocysteine is an amino acid homolog of the amino acid cysteine, differing by having an additional methylene $\left(-\mathrm{CH}_{2}-\right)$ group. The S-adenyl homocysteine (SAH) produced (Fig. 1) from demethylation of S-adenosylmethionine is usually quickly transformed into homocysteine, which is more stable than SAH.

High plasma homocysteine has been shown to compromise the blood-brain barrier in mice. ${ }^{3}$ Homocysteine promotes atherosclerosis (Fig. 2) through fibrin deposition, oxidant stress, cytokine release, inflammation, and other mechanisms. ${ }^{4}$ The atherosclerosis associated with high plasma homocysteine may also be partly due to homocysteine-induced stress to the endoplasmic reticulum of endothelial cells. ${ }^{5}$ Homocysteine damages endothelial cells. ${ }^{6}$ Endothelial dysfunction has been shown to incrementally increase with incrementally higher



Fig. 2: Vascular compromise by homocysteine 


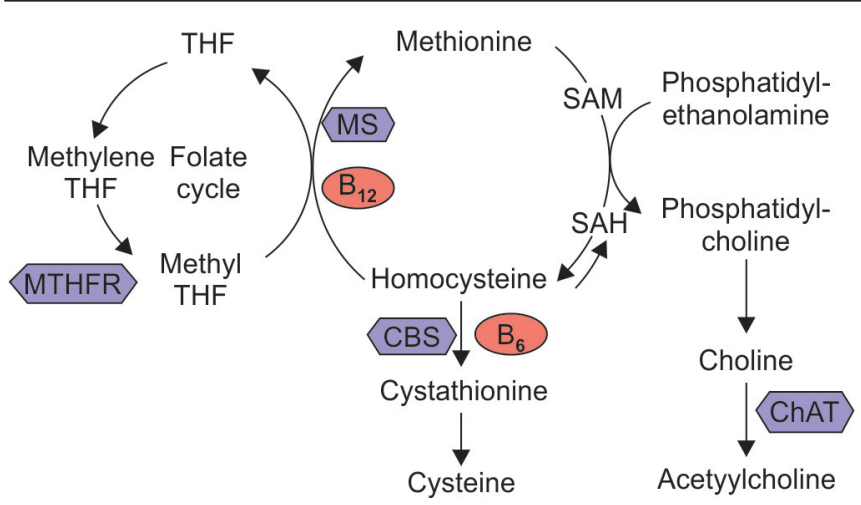

Fig. 3: Folate and methionine cycle showing the site of action of $\mathrm{B}_{6}$ and $\mathrm{B}_{12}$

doses of oral methionine (and subsequent incrementally higher plasma homocysteine) in normal human subjects.? Although B vitamin therapy has been shown to reduce plasma homocysteine in clinical trials, little or no reduction in cardiovascular disease risk has been seen ${ }^{8,9}$ (Fig. 3).

Women with AT III deficiency are prone to pregnancyassociated venous thromboembolism. Yamada et $\mathrm{al}^{10}$ have reported two cases with such deficiencies, which were genetically confirmed, in whom the pregnancies were successfully managed with prophylactic therapies for thrombosis.

Obstetrician should consider:

- Therapeutically anticoagulating these patients postpartum,

- Prophylactic anticoagulation throughout pregnancy, especially in patients with a history of thrombosis,

- Assaying AT III in plasma rather than serum, and

- Prepregnancy counseling, including information about the autosomal dominant inheritance of hereditary AT III deficiency. ${ }^{11}$

\section{CONCLUSION}

Thrombophilia is associated with increased frequency of late pregnancy wastage, and hyperhomocysteinemia should be identified in women with RPL, because therapeutic normalization might permit a normal birth.

\section{REFERENCES}

1. Sarig G, Younis JS, Hoffman R, Lanir N, Blumenfeld Z, Brenner B. Thrombophilia-associated pregnancy loss. Fertil Steril 2002;77:342-347.

2. Brenner B, Hoffman R, Blumenfeld Z, Weiner Z, Younis JS. Gestational outcome in thrombophilic women with recurrent pregnancy loss treated by enoxaparin. Thromb Haemost 2000 May;83(5):693-697.

3. Kamath AF, Chauhan AK, Kisucka J, Dole VS, Loscalzo J, Handy DE, Wagner DD. Elevated levels of homocysteine compromise blood-brain barrier integrity in mice. Blood 2006 Jan;107(2):591-593.

4. McCully KS. Homocysteine, vitamins, and vascular disease prevention. Am J Clin Nutr 2007 Nov;86(5):1563S-1568S.

5. Majchrzak D, Singer I, Männer M, Rust P, Genser D, Wagner KH, Elmadfa I. B-vitamin status and concentrations of homocysteine in Austrian omnivores, vegetarians and vegans. Ann Nutr Metab 2006 Sep;50(6):485-491.

6. Werstuck GH, Lentz SR, Dayal S, Hossain GS, Sood SK, Shi YY, Zhou J, Maeda N, Krisans SK, Malinow MR, et al. Homocysteine-induced endoplasmic reticulum stress causes dysregulation of the cholesterol and triglyceride biosynthetic pathways. J Clin Invest 2001 May;107(10):1263-1273.

7. Chambers JC, Obeid OA, Kooner JS. Physiological increments in plasma homocysteine induce vascular endothelial dysfunction in normal human subjects. Arterioscler Thromb Vasc Biol 1999 Dec;19(12):2922-2927.

8. Maron Ba, Loscalzo J. The treatment of hyperhomocysteinemia. Ann Rev Med 2009 Feb;60:39-54.

9. Lin CP, Chen YH, Leu HB, Lin SJ, Chen YL, Huang SL, Chen JW. Anti-inflammatory strategies for homocysteine-related cardiovascular disease. Front Biosci 2009 Jan;14:3836-3845.

10. Yamada T, Yamada H, Morikawa M, Kato EH, Kishida T, Ohnaka Y, Nikaido H, Ozawa T, Fujimoto S. Management of pregnancy with congenital antithrombin III deficiency: two case reports and a review of the literature. J Obstet Gynaecol Res 2001 Aug;27(4):189-197.

11. Nelson DM, Stempel LE, Brandt JT. Hereditary antithrombin III deficiency and pregnancy: report of two cases and review of the literature. Obstet Gynecol 1985 Jun;65(6):848-853. 